\title{
Use of direct oral anticoagulants for the prevention and treatment of thromboembolic disease in patients with reduced renal function: a short review of the clinical evidence
}

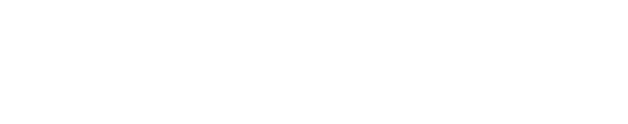

\author{
Kristine C Willett \\ Amanda M Morrill \\ Department of Pharmacy Practice, \\ School of Pharmacy, MCPHS \\ University, Manchester, NH, USA
}

\begin{abstract}
Background: The use of direct oral anticoagulants (DOACs) is restricted by the limitations of clinical trials guiding therapy for patients with renal impairment, as many of these trials excluded patients with severe renal impairment. There are currently four agents available: dabigatran, rivaroxaban, apixaban, and edoxaban. The purpose of this review was to 1) describe current recommended dosing for each DOAC and published postmarketing data, including case reports, on the use of these agents in the renally impaired; and 2) discuss patient adherence and satisfaction and the cost of these agents.
\end{abstract}

Materials and methods: A literature search was conducted using Medline and Embase with the terms "dabigatran" or "rivaroxaban" or "apixaban" or "edoxaban" and "renal impairment". Clinical trials and case reports describing the use of DOAC therapy in patients with renal impairment were reviewed. A second search was conducted to find articles evaluating patient adherence, patient satisfaction, and pharmacoeconomics of DOACs.

Results: There are a multitude of subgroup and post hoc analyses, as well as six case reports with dabigatran and one case report with apixaban, that provide insight for the clinical use of DOACs in patients with renal impairment. Dabigatran exhibits the greatest level of renal elimination, and there are data from clinical trials and several case reports that warrant reconsideration before use. Other DOACs may be a better option in patients with impaired renal function. Further, data from patient-adherence studies have suggested that DOACs that are dosed daily (rather than twice daily) are optimal and preferred. There does not appear to be a cost difference between DOACs and warfarin therapy.

Conclusion: DOAC therapy in patients with impaired renal function requires more critical review of study data, as these patients may have increased risk of bleeding. It is also valuable to consider patient preferences and cost when selecting the appropriate option for oral anticoagulation.

Keywords: anticoagulation, dabigatran, rivaroxaban, apixaban, edoxaban, renal impairment, adherence, cost

\section{Introduction}

Since 2010, there has been a surge in direct oral anticoagulants (DOACs) approved for prevention and treatment of thromboembolic disorders. Currently, there are four available agents, including a direct thrombin inhibitor - dabigatran (Pradaxa ${ }^{\circledR}$ ) - as well as three factor-Xa inhibitors: rivaroxaban (Xarelto ${ }^{\circledR}$ ), apixaban (Eliquis ${ }^{\circledR}$ ), and edoxaban (Savaysa $\left.{ }^{\circledR}\right)$. These agents are all US Food and Drug Administration (FDA) approved for long-term anticoagulation management in patients with nonvalvular atrial
Correspondence: Kristine C Willett Department of Pharmacy Practice, School of Pharmacy, MCPHS University, 1260 Elm Street, Manchester, NH 03I0I, USA Tel +l 6033141778

Email kristine.willett@mcphs.edu 
fibrillation (NVAF) and treatment of venous thromboembolism (VTE). ${ }^{1-4}$ Dabigatran, rivaroxaban, and apixaban have also been approved for prevention of recurrent VTE and prophylaxis of VTE in patients who have undergone hipreplacement surgery. ${ }^{1-3}$ Notably, rivaroxaban and apixaban have approval for hip- and knee-surgery patients. ${ }^{2,3}$ Given the increasingly wide range of patients eligible for DOAC therapy, there is added value to understanding their roles in special populations, particularly those with renal impairment.

The advantage that DOACs provide over other OACs is their predictable pharmacokinetic (PK) and pharmacodynamic (PD) profiles in healthy patients. As such, there is less monitoring required for determination of efficacy. A summary of DOAC PK profiles can be found in Table 1. Despite the decreased monitoring and need for dosage adjustments, alterations in PK and PD properties in patients with renal impairment necessitate further evaluation. It is estimated that chronic kidney disease (CKD) has an incidence of 26 million patients in the US. Further, it should be noted that renal impairment independently confers an increased risk of bleeding, regardless of the AC utilized. In 2009, Go et al had information from a large database published, which reported that impaired renal function is a risk factor for ischemic stroke or VTE. ${ }^{5}$ Furthermore, clinical studies leading to the approval of DOACs excluded patients with severe renal impairment, defined as a creatinine clearance $(\mathrm{CrCl})$ less than $30 \mathrm{~mL} / \mathrm{min}$. Renal function in these studies were based upon the Cockcroft-Gault (CG) equation. While dosing recommendations for DOAC therapy are based on $\mathrm{CrCl}$ calculated using the $\mathrm{CG}$ equation, patient-specific factors, such as age, hydration status, and other comorbidities, may result in fluctuations in serum $\mathrm{Cr}(\mathrm{SCr})$. This should be considered when deciding on optimal therapy for patients.

\section{Pharmacology of DOACs}

Dabigatran etexilate is metabolized into an active metabolite and eliminated through glomerular filtration (GF; 80\%). ${ }^{1,6}$
The FDA-approved dose of dabigatran is $150 \mathrm{mg}$ twice daily for patients with $\mathrm{CrCl}$ greater than $30 \mathrm{~mL} / \mathrm{min}$ and a reduction to $75 \mathrm{mg}$ twice daily in those with impaired renal function. ${ }^{1}$ It is known that the half-life of dabigatran increases from 13 hours to 27 hours in patients with severe renal impairment, though manufacturer dosing recommendations were extrapolated from results of subgroup or post hoc analyses, rather than from clinical evaluation. ${ }^{6}$ Data from Phase I, II, and III studies led to an FDA-approved dose of dabigatran of $75 \mathrm{mg}$ twice daily for patients with $\mathrm{CrCl}$ less than $30 \mathrm{~mL} / \mathrm{min}$ (150 $\mathrm{mg}$ twice daily for normal renal function). ${ }^{1}$ Lehr et al performed PK dose identification using computer models, which confirmed that the $75 \mathrm{mg}$ twice-daily dose is safe and effective in patients with impaired renal function, though it is important to note that this dose was not used in clinical studies. $^{7}$

Rivaroxaban is FDA approved to manage NVAF patients requiring anticoagulation at a dose of $20 \mathrm{mg}$ daily and for treatment of VTE at $15 \mathrm{mg}$ twice daily for 21 days, followed by $20 \mathrm{mg}$ daily. ${ }^{2}$ The clearance of rivaroxaban is approximately 33\% renal. A PK and PD assessment of one $10 \mathrm{mg}$ dose in patients with varying degrees of renal impairment found that reduced renal function results in significant reduction in rivaroxaban clearance $(P<0.001) .{ }^{8}$ Given the alterations in renal clearance, clinical trials leading to the approval of rivaroxaban excluded patients with a $\mathrm{CrCl}$ less than $30 \mathrm{~mL} / \mathrm{min} .{ }^{9,10}$ Therefore, manufacturer recommendations suggest avoidance of rivaroxaban in these patients.

Apixaban is FDA approved for NVAF and treatment of VTE, with a recommended dose of $5 \mathrm{mg}$ twice daily. ${ }^{3}$ While there is no dosage adjustment necessary for renal impairment, patients who meet two of the following criteria receive a reduced dose of $2.5 \mathrm{mg}$ twice daily: $\mathrm{SCr}$ greater than $1.5 \mathrm{mg} / \mathrm{dL}$, older than 80 years, or weight less than $60 \mathrm{~kg}$. Of note, studies evaluating apixaban in patients with NVAF for prevention of stroke have excluded patients with severe renal disease, which was defined as $\mathrm{CrCl}$ less than $25 \mathrm{~mL} / \mathrm{min}$ or

Table I Pharmacokinetic profiles for direct oral anticoagulants in patients with normal renal function

\begin{tabular}{|c|c|c|c|c|}
\hline & Absorption & Distribution & Metabolism & Excretion \\
\hline Dabigatran & $\begin{array}{l}F=3 \%-7 \% ; T_{\max }=I \text { hour (may be delayed } \\
\text { for up to } 2 \text { hours when taken with a meal) }\end{array}$ & PPB: $35 \%$ & Conjugation & $t_{1 / 2}=12-17$ hours; renal $(80 \%)$ \\
\hline Rivaroxaban & $\begin{array}{l}F=80 \% ; T_{\max }=2-4 \text { hours; coadministration } \\
\text { with food increases } F\end{array}$ & PPB: $92 \%-95 \%$ & Oxidation by CYP $3 A 4 / 5$ and $2 \mathrm{~J} 2$ & Fecal (2I\%); renal (30\%) \\
\hline Apixaban & $F=50 \% ; T_{\max }=3-4$ hours & PPB: $87 \%$ & $\begin{array}{l}\text { Oxidation by CYP } 3 \mathrm{~A} 4 \text { (major); } \\
\text { IA } 2,2 \mathrm{C} 8,2 \mathrm{C} 9,2 \mathrm{CI} 9,2 \mathrm{~J} 2 \text { (minor) }\end{array}$ & $t_{1 / 2}=10-14 \mathrm{hrs} ;$ renal $(27 \%)$ \\
\hline Edoxaban & $\begin{array}{l}F=62 \% ; T_{\max }=I-2 \text { hours; absorption not } \\
\text { affected by food }\end{array}$ & PPB: $55 \%$ & $\begin{array}{l}\text { Hydrolysis; conjugation; oxidation } \\
\text { by CYP 3A4 }\end{array}$ & $\begin{array}{l}t_{1 / 2}=10-14 \text { hours; fecal }(60 \%) ; \\
\text { renal }(35 \%)\end{array}$ \\
\hline
\end{tabular}

Note: Data from previous studies. ${ }^{6,7,46}$

Abbreviations: $T_{\max }$, time to maximum concentration; PPB, plasma protein binding; $t_{1 / 2}$, half-life; $F$, bioavailability. 
$\mathrm{SCr}$ greater than $2.5 \mathrm{mg} / \mathrm{dL} .{ }^{11,12}$ Hohnloser et al conducted a subgroup analysis from ARISTOTLE, which evaluated the outcomes of stroke and systemic embolism and baseline renal function. Despite the exclusion of patients with severe renal impairment, the risk of major bleeding remained higher in those with greater impairment, stratifying those with $\mathrm{CrCl}>80 \mathrm{~mL} / \mathrm{min}$, those with $\mathrm{CrCl}$ 50-79 $\mathrm{mL} / \mathrm{min}$, and those with $\mathrm{CrCl}<50$. Risk of bleeding, ischemic stroke, and all-cause mortality were greatest in the group with the most impairment in renal function. ${ }^{13}$

Edoxaban is approximately $50 \%$ renally excreted. Interestingly, NVAF patients with $\mathrm{CrCl}>95 \mathrm{~mL} / \mathrm{min}$ should not take edoxaban, as an increased risk of thromboembolic events has been found. ${ }^{4}$ As with studies of dabigatran and rivaroxaban, edoxaban studies have excluded patients with $\mathrm{CrCl}<30 \mathrm{~mL} / \mathrm{min}$ and also reduced doses by $50 \%$ in patients with $\mathrm{CrCl}$ of $30-50 \mathrm{~mL} / \mathrm{min}^{14}$ The purpose of this review is to 1) describe current recommended dosing for each DOAC and discuss published postmarketing data, including case reports, on the use of these agents in the renally impaired, and 2) discuss patient adherence, patient satisfaction, and cost of these agents.

\section{Materials and methods}

A literature search was conducted using Medline (December 1946 to week 1, 2016) and Embase (1980 to week 2, 2017) with the terms "dabigatran" or "rivaroxaban" or "apixaban" or "edoxaban" and "renal impairment". Clinical trials evaluating DOAC therapy in renally impaired patients since their initial FDA approval were reviewed. Case reports and case series reporting on use of DOACs for patients with renal impairment were also included. A second search was conducted to find articles evaluating patient adherence, patient satisfaction, and pharmacoeconomics. Results of these searches were included for review.

\section{Results \\ Dabigatran}

The RE-LY study was conducted to evaluate the efficacy and safety of dabigatran etexilate in 18,113 patients with NVAF requiring chronic anticoagulation therapy. ${ }^{15}$ This doubleblind multicenter study randomized patients into one of three treatment arms (dabigatran $150 \mathrm{mg}$ twice daily, dabigatran $110 \mathrm{mg}$ twice daily, or warfarin adjusted to achieve an international normalized ratio [INR] of 2-3). Renal function was monitored at 3, 6, and 12 months, and annually until study completion. Notably, in patients with $\mathrm{CrCl}$ less than or equal to $30 \mathrm{~mL} / \mathrm{min}$, therapy was held until $\mathrm{CrCl}$ increased to greater than $30 \mathrm{~mL} / \mathrm{min}$. The population evaluated in RE-LY had average age of 71 years. Results concluded that both doses of dabigatran were noninferior to warfarin therapy, but the $150 \mathrm{mg}$ twice-daily group had significantly fewer strokes or less systemic embolism compared to the $110 \mathrm{mg}$ group $(P<0.001)$. The dabigatran $150 \mathrm{mg}$ group had a trend toward greater risk of major bleeding compared to the $110 \mathrm{mg}$ group $(P=0.052)$. Following the results of RE-LY, Hijazi et al further analyzed the effect of baseline renal function on the primary outcome of stroke or systemic embolism. ${ }^{16}$ Interestingly, investigators reported that there was a decreased risk of the primary outcome in patients with greater calculated $\mathrm{CrCl}$. That is, patients with baseline $\mathrm{CrCl}$ greater than or equal to $80 \mathrm{~mL} / \mathrm{min}$ had an incidence of stroke or systemic embolism of $0.88 \%$ compared to $1.59 \%$ in patients with $\mathrm{CrCl}$ of $50-79 \mathrm{~mL} / \mathrm{min}$ and $2.16 \%$ in those with $\mathrm{CrCl}$ less than $50 \mathrm{~mL} / \mathrm{min}$.

Further, Böhm et al conducted a post hoc analysis from the RE-LY study to assess overall changes in renal function in all 18,113 patients for up to 30 months. ${ }^{17}$ A review of baseline characteristics revealed that $18.6 \%$ of patients had moderate renal impairment $(\mathrm{CrCl} 30-50 \mathrm{~mL} / \mathrm{min})$, and $36.6 \%$ met the classification for stage $3 \mathrm{CKD}$. Investigators noted that a decline in renal function occurred in all three treatment arms, and was significantly greater in the warfarin group compared to the dabigatran $110 \mathrm{mg}$ twice-daily $(P=0.0009)$ and dabigatran $150 \mathrm{mg}$ twice-daily $(P=0.0002)$ groups. As such, patients taking warfarin were more likely to discontinue therapy then those taking dabigatran $110 \mathrm{mg}$ and $150 \mathrm{mg}$ ( $P=0.0032$ and $P=0.0014$, respectively). A multivariate analysis was conducted, and found that the comedications with the greatest risk of worsening renal function included angiotensin-receptor blockers, amiodarone, and diuretics. Also, elderly patients and diabetic patients were more likely to have renal function decline.

The aforementioned studies assessed changes in renal function without correlation with clinical implications of the use of dabigatran in renal impairment. There have been seven published case reports of dabigatran-associated adverse events in patients with $\mathrm{CrCl}$ less than $30 \mathrm{~mL} / \mathrm{min} .{ }^{18-24}$ Notably, all seven of these reports included patients taking dabigatran $150 \mathrm{mg}$ twice daily. Five of the six reports showed gastrointestinal ulcers or bleeding in patients aged 66-92 years. Bleeding occurred in one patient, a 92-year-old man, after a single dose and unfortunately resulted in fatality. ${ }^{19}$ In another case, a 79-year-old man presented with acute renal failure after 6 weeks of dabigatran therapy. ${ }^{22}$ Worsening renal function was found during a 6-week follow-up, and renal Doppler detected the presence of renal artery stenosis. 
Dabigatran was discontinued, and the patient remains on longterm hemodialysis. A similar case report was described in a 58-year-old woman with a calculated $\mathrm{CrCl}$ of $16.9 \mathrm{~mL} / \mathrm{min} .{ }^{21}$ Notably, the woman presented with epistaxis and hematochezia secondary to renal failure and required two doses of idarucizumab to achieve anticoagulation reversal. For a description of published dabigatran studies, see Table 2.

There have been limited studies directly evaluating the efficacy and safety of DOACs in the renally impaired. However, an open-label prospective observational study published in January 2017 evaluated patients with moderate renal impairment $(\mathrm{CrCl} 30-50 \mathrm{~mL} / \mathrm{min})$ undergoing total hip or knee replacement. ${ }^{25}$ The primary outcome was incidence of major bleeding events, but incidence of VTE and allcause mortality were also evaluated. A total of 428 patients with a median age of 80 years were enrolled. The majority of patients were female (75.7\%) and had an average $\mathrm{CrCl}$ of $42.5 \mathrm{~mL} / \mathrm{min}$. The overall incidence of major bleeding was $2.1 \%$. Although there were no cases of fatal bleeding events, the incidence was higher than that reported from a rivaroxaban study $(0.4 \%)$ evaluating its use in orthopedic patients. ${ }^{26}$ The difference in incidence of major bleeding can be attributed to impairment of renal function, advanced age, and greater comorbid conditions in the dabigatran observational study. The overall incidence of VTE and all-cause mortality was $0.7 \%(n=3)$, which is comparable to other large observational DOAC studies. ${ }^{25}$

\section{Rivaroxaban}

The majority of available data on the use of rivaroxaban in patients with renal impairment comes from subgroup analyses of major Phase III clinical trials in the US and Japan. ${ }^{27,28}$ A subgroup analysis of ROCKET-AF (a multicenter, randomized, double-blind, double-dummy, event-driven trial comparing the efficacy of rivaroxaban to warfarin as thromboprophylaxis in patients with NVAF) assessed if worsening renal function affected study outcomes. ${ }^{27}$ Patients were included in the subanalysis if they had at least one SCr measurement after baseline assessment. Worsening renal function, defined as $>20 \%$ decrease in $\mathrm{CrCl}$ from baseline, calculated using the $\mathrm{CG}$ equation, was used as a time-dependent variable in the Cox proportional-hazard model. Patients with $\mathrm{CrCl}$ of $50 \mathrm{~mL} /$ min or greater received rivaroxaban $20 \mathrm{mg}$ daily, and those with $\mathrm{CrCl} 30-50 \mathrm{~mL} / \mathrm{min}$ received the lower $15 \mathrm{mg}$ dose. Patients were excluded from the main trial if they had $\mathrm{CrCl}$ $<30 \mathrm{~mL} / \mathrm{min}$, and treatment was discontinued in those with two consecutive $\mathrm{CrCl}$ measurements $<25 \mathrm{~mL} / \mathrm{min}$ during follow-up readings. There were similar baseline characteristics between the rivaroxaban and warfarin groups, and overall there was no difference in rates of worsening renal function. A total of 62 participants discontinued therapy due to renal failure; of these, 35 were in the rivaroxaban group. There was no difference in incidence of stroke, systemic embolism, and bleeding between those patients with stable or worsening renal function. However, regardless of treatment, the worsening renal function group had had a higher rate of vascular death (2.21 versus 1.41 events per 100 patient-years, $P=0.026)$ and all-cause mortality (3.1 versus 1.93 events per 100 patient-years, $P=0.0067$ ) compared to those with stable renal function. The composite end point of stroke, systemic embolism, vascular death, or myocardial infarction occurred significantly more in the worsening renal function group (hazard ratio $1.4, P=0.0023$ ).

Interestingly, in rivaroxaban patients with worsening renal function, there were 1.93 more gastrointestinal bleeds per 100 patient-years $(P=0.02)$, but there was no difference in major or nonmajor clinically relevant bleeding compared to warfarin patients. However, there was also less incidence of

Table 2 Published dabigatran case reports

\begin{tabular}{|c|c|c|c|c|c|}
\hline Case report & Age, years & $\mathrm{CrCl}(\mathrm{mL} / \mathrm{min})$ & Dabigatran dose; duration & Indication & Outcome \\
\hline Fountzilas et $\mathrm{al}^{18}$ & 82 & $\mathrm{NR} ; \mathrm{SCr} 1.78 \mathrm{mg} / \mathrm{dL}$ & I50 mg BID; I week & NVAF & $\begin{array}{l}\text { Hospitalized for weakness, dizziness, } \\
\text { nausea, and vomiting; INR =7.25 }\end{array}$ \\
\hline Kernan et $\mathrm{al}^{19}$ & 92 & 24.2 & I50 mg; single dose & NVAF & Hospitalization for $\mathrm{GiB}$ \\
\hline Maddry et $\mathrm{al}^{20}$ & 74 & $\mathrm{NR} ; \mathrm{SCr} 3.1 \mathrm{mg} / \mathrm{dL}$ & I50 mg BID; NR & NVAF & Fatal GiB \\
\hline Marino et $\mathrm{al}^{21}$ & 58 & $\mathrm{NR} ; \mathrm{SCr} 2.6 \mathrm{mg} / \mathrm{dL}$ & 150 mg BID; 3 months & NVAF & $\begin{array}{l}\text { Hospitalization; ARF; patient developed } \\
\text { ESRD and remains on hemodialysis }\end{array}$ \\
\hline Shafi et $\mathrm{a}^{22}$ & 79 & $27.2^{\mathrm{a}}$ & 150 mg BID; 6 weeks & NVAF & $\begin{array}{l}\text { ARF requiring hemodialysis; two doses } \\
\text { of idarucizumab were administered }\end{array}$ \\
\hline Stöllberger et $\mathrm{a}^{23}$ & 83 & 21 & 150 mg BID; 4 weeks & NVAF & Hospitalization for GiB \\
\hline Wychowski and Kouides ${ }^{24}$ & 66 & 15 & I50 mg BID; 8 weeks & NVAF & Hospitalization for GiB \\
\hline
\end{tabular}

Note: ${ }^{a}$ Calculated using eGFR.

Abbreviations: $\mathrm{CrCl}$, creatinine clearance; NR, not reported; SCr, serum creatinine; BID, bis in die (twice daily); NVAF, nonvalvular atrial fibrillation; INR, international normalized ratio; GiB, gastrointestinal bleeding; ARF, acute renal failure; ESRD, end-stage renal disease; eGFR, estimated glomerular filtration rate. 
stroke or systemic embolism in the worsening renal function group taking rivaroxaban compared to the worsening renal function group taking warfarin ( $P=0.05$ for interaction). This finding was not seen in patients with stable renal function. When a sensitivity analysis was performed with the MDRD and CKD-EPI equations, results were mostly similar.

The J-ROCKET AF study assessed similar outcomes to ROCKET-AF, but took place in Japan and used lower doses of rivaroxaban compared to the US dosing. ${ }^{28}$ Patients received rivaroxaban $15 \mathrm{mg}$ daily or $10 \mathrm{mg}$ daily for $\mathrm{CrCl}$ $30-40 \mathrm{~mL} / \mathrm{min}$. Of note, patients older than 70 years also had a lower target INR of 1.6-2.6. A subanalysis of J-ROCKET AF investigated the effect of age (greater than or less than 75 years) on study outcomes, and stratified results based on renal function in the elderly group. Approximately $30 \%$ more patients in the 75-year-and-older group had $\mathrm{CrCl}$ less than $50 \mathrm{~mL} / \mathrm{min}$ compared to the younger cohort; this group also had lower body weight. The primary safety outcome (major and nonmajor clinically relevant bleeding) occurred more in the rivaroxaban group in patients with moderate renal dysfunction, but was not significant. Conversely, the primary efficacy outcomes occurred less in the rivaroxaban group in patients with moderate renal dysfunction, though this was not significant. There was no significant difference between normal and impaired renal function for either of the efficacy or safety outcomes. The lower target INR of the J-ROCKET study may make it difficult to extrapolate results to the US population. More comprehensive assessment of the effect of renal function on rivaroxaban efficacy in the setting of CKD will be further elucidated in the X-NOAC study, a randomized controlled multicenter trial in Japan. ${ }^{29}$

In addition to these subgroup analyses, there is one published case report from Austria of an 82-year-old woman with a history of NVAF treated with rivaroxaban, which appeared to be ineffective. ${ }^{30}$ The patient was given rivaroxaban therapy at $10 \mathrm{mg}$ daily after hospitalization for ischemic stroke, and it was reported that she was unable to achieve stable INR with warfarin therapy. Eleven days after initiation, she was rehospitalized with signs of recurrent stroke. $\mathrm{Her} \mathrm{CrCl}$ at the time of initial stroke was $32 \mathrm{~mL} / \mathrm{min}$, resulting in off-label dosage adjustment to $10 \mathrm{mg}$ daily. This reduced dose is likely ineffective, despite poor renal function. In appropriate use of rivaroxaban or any DOAC therapy should be limited until there are more clinical data available.

\section{Apixaban}

Data on apixaban are limited to a prespecified secondary subanalysis of the ARISTOTLE trial, which evaluated primary efficacy (stroke or systemic embolism) and safety (major bleeding) outcomes in relation to renal function, and one published case report. ${ }^{13,31}$ In the ARISTOTLE trial, renal function was assessed primarily using the CG equation, but was also with the CKD-EPI equation and cystatin C measurement. ${ }^{13}$ The standard apixaban dose was $5 \mathrm{mg}$ twice daily, and $2.5 \mathrm{mg}$ twice daily for patients meeting two of the aforementioned characteristics. An inverse relationship was identified between renal function and annualized stroke risk, as well as $\mathrm{CrCl}$ and all-cause mortality, which was three times higher in the estimated GF rate $(\mathrm{eGFR})<50 \mathrm{~mL} / \mathrm{min}$ group compared to the $>80 \mathrm{~mL} / \mathrm{min}$ group. The relationship between renal function and cardiovascular events was consistent, regardless of estimation method. For the primary efficacy outcome, apixaban was superior to warfarin independently of renal function and method of calculation. Apixaban patients had lower rates of major bleeding compared to warfarin, regardless of renal function. Because patients with greater renal impairment were more likely to have the lower dose, in an additional subanalysis of bleeding rates in only those patients on the $5 \mathrm{mg}$ dose, the direct relationship remained statistically significant. A subanalysis of AVERROES evaluated the use of apixaban 2.5 or $5 \mathrm{mg}$ twice daily in patients with stage 3 CKD compared to $81-324 \mathrm{mg}$ of aspirin daily for thromboprophylaxis in AF. ${ }^{32}$ The criteria for the $2.5 \mathrm{mg}$ dose of apixaban was the same as the ARISTOTLE trial.

Patients selected for this trial had previously failed or were not candidates for warfarin therapy, and eGFR was calculated using the CKD-EPI equation. Stroke or systemic embolism, the primary efficacy end point, occurred more frequently in patients with stage $3 \mathrm{CKD}$ compared to those with higher renal function. Notably, compared with patients with an eGFR of $60 \mathrm{~mL} / \mathrm{min}$ or higher, patients with stage 3 $\mathrm{CKD}$ had significantly higher $\mathrm{CHADS}_{2}$ scores. Unsurprisingly, these patients were also older, had a higher incidence of past stroke or TIA, and more hypertension, diabetes, and heart failure. There was a direct relationship between $\mathrm{CHADS}_{2}$ score and rate of primary efficacy outcome and hemorrhage in the aspirin group. However, in the stage 3 CKD group of patients taking apixaban, there was a direct relationship between $\mathrm{CHADS}_{2}$ score and the primary efficacy outcome and death, but not hemorrhage. In both the aspirin and apixaban groups, there was an inverse relationship identified between eGFR and hemorrhage rate.

There has been one published apixaban case report in a renally impaired patient, which unfortunately resulted in fatal gastrointestinal bleeding. ${ }^{31}$ The patient was a 62-year-old woman with end-stage renal disease requiring hemodialysis, 
and was admitted for calciphylaxis secondary to warfarin therapy. Her history of NVAF required her to take warfarin, which was changed to apixaban $2.5 \mathrm{mg}$ twice daily based upon manufacturer prescribing recommendations. After eleven doses, she developed clinical signs of bleeding and was found to have an anti-factor Xa level of greater than $2 \mathrm{IU} / \mathrm{mL}$. Ultimately, the patient died of complications, which did not implicate anticoagulation as the primary cause. Notably, patients with end-stage renal disease were excluded from the ARISTOTLE trial; the trial did not enroll patients with $\mathrm{CrCl}<25 \mathrm{~mL} / \mathrm{min}$.

\section{Edoxaban}

Koretsune et al conducted a short-term (12-week) safety analysis of edoxaban $15 \mathrm{mg}$ daily in NVAF Japanese patients with severe renal impairment, which was defined as $\mathrm{CrCl}$ 15-29 $\mathrm{mL} / \mathrm{min}^{33}$ This was an open-label study, which randomized 93 patients with severe renal impairment to receive $15 \mathrm{mg}$ daily, and patients with normal or moderately impaired renal function received either 30 or $60 \mathrm{mg}$ of edoxaban. There were no major bleeding events in any of the treatment groups. There was one case of clinical relevant nonmajor bleeding in the $60 \mathrm{mg}$ group, and all bleeding events in patients with several renal impairment were minor events.

\section{Adherence}

Unlike warfarin, two DOACs - apixaban and dabigatran etexilate - are dosed twice daily. Numerous studies investigating the relationship between dosing frequency and medication adherence have been completed, and most found increased adherence with once-daily dosing. ${ }^{34-36}$ A systematic review of 51 prospective studies comparing once-, twice-, three-time-, and four-time-daily dosing regimens assessed using electronic monitoring (EM) found that patients with chronic disease were significantly more adherent to oncedaily dosing compared to the other regimens. ${ }^{34}$ Adherence for taking medication was $6.7 \%$ lower in twice-daily dosing compared to once daily. A smaller meta-analysis of 29 prospective studies focusing on adherence to cardiovascular medications found similar results. Similarly, adherence was measured using EM, and adherence was 6.9\% lower for twice-daily dosing regimens compared to once daily $(P<0.01) .{ }^{35}$ Furthermore, $22.9 \%$ less doses were taken on time in the twice-daily regimens $(P<0.01)$. Conversely, a meta-analysis of 76 studies assessing dosing adherence using EM found lower adherence in twice-daily dosing, but it was not statistically significant. Unlike the previously discussed studies, the regimens were for both acute and chronic disease states. ${ }^{36}$ Similarly, once-daily dosing adherence was significantly higher than three- and four-time-daily dosing in this study as well ( $P=0.008$ and $P<0.001$, respectively). Limited data specific to patient adherence to DOACs are available. A retrospective cohort study in 5,376 patients in the Veterans Affairs health care system assessed adherence to dabigatran, apixaban, and rivaroxaban for NVAF in the first year of therapy by estimating proportion of days covered. ${ }^{37}$ The study found that $27.8 \%$ of patients were non-adherent (defined as proportion of days covered $<0.8$ ).

Even with the potential benefit of once-daily dosing in regard to adherence, apixaban and edoxaban have much shorter half-lives (12 and 10-14 hours, respectively) compared to warfarin's half-life of 21-89 hours., ${ }^{3,438}$ That is, if a dose is missed, the patient remains undercoagulated and at risk of a thrombotic event. Conversely, due to the longer duration of action of warfarin, a patient who misses a dose may remain within the therapeutic range with no further action required. Even in the most adherent once-daily dosingtreatment group in the studies described, the average rate of nonadherence remains at $7 \%$, which may still place patients at risk of an event. Furthermore, the use of EM is not standard in practice, and may result in an overestimation of adherence compared to "real-world" patients.

\section{Patient satisfaction}

Since the approval of DOACs, one of the major marketing points by drug companies has been patient satisfaction with decreased monitoring and dietary ramifications of these new agents compared to warfarin. Most of the studies have focused on patients' willingness to switch from warfarin or their satisfaction with dabigatran, which is not surprising, considering the other agents are relatively newer to the market. The SWITCH survey assessed the willingness of 180 patients enrolled at a warfarin clinic to change therapies from warfarin to dabigatran utilizing a written survey tool. ${ }^{39}$ Overall, $80 \%$ of respondents reported satisfaction with therapy, but 58\% of respondents were willing to change ACs. Interestingly, patients older than 70 years were significantly more willing to switch compared to those younger than 70 years $(P=0.017)$. Factors contributing to dissatisfaction with warfarin included frequent provider visits, and the most common factor that would deter a patient from switching was cost. Similar results were reported by a prospective observational study surveying 260 patients on warfarin therapy. ${ }^{40}$ In this study, additional reasons supporting willingness to switch included fewer dietary restrictions and equal efficacy. In a small study in Australia, 76 patients with and without AF were surveyed to identify factors influencing preference for anticoagulation therapy. ${ }^{41}$ Unlike the two aforementioned studies, frequency 
of blood tests, along with dosing frequency and drug-food interactions, were considered less important than efficacy and safety. Likewise, cost was of high importance, and the affinity for newer agents increased as cost decreased.

Although limited, there are studies assessing patient opinions on the medication they were currently taking without the option to switch or make a comparison. Coleman et al completed a cross-sectional study assessing patient perspective on medication burden. ${ }^{42}$ The self-administered survey of 80 patients with AF on warfarin $(n=65)$ or nonwarfarin $(n=15)$ found that patients on warfarin had less favorable feelings toward their anticoagulation regimen using the Anti-Clot Treatment Scale and Benefit and Burden subscale. Notably, there was no difference in benefit scores, but the warfarin group perceived a higher burden due to their medication. These results may not accurately reflect the entirety of patients on DOACs, as only two patients in the nonwarfarin group were on dabigatran and the remainder were taking antiplatelet agents alone or in combination. In a subgroup analysis of RE-LY, no difference was found in health-related quality of life between patients being treated with warfarin and those being treated with dabigatran for 1 year. ${ }^{43}$ Additionally, there was no difference in either group from baseline to the end of the study. The XANTUS study, the first longterm prospective observational study of rivaroxaban use in an international population of patients with AF, followed 6,784 patients in Europe, Israel, and Canada prescribed rivaroxaban for NVAF for up to 1 year or at least 30 days after discontinuation. ${ }^{44}$ Although the primary objective was to assess safety and efficacy, other measured outcomes included patient satisfaction with treatment based on a standardized questionnaire. This indicated that $75.1 \%$ of patients reported being satisfied or very satisfied with therapy. This correlated with medication persistence of $80 \%$ for 1 year. For those who discontinued, the most common reason was adverse effects.

\section{Pharmacoeconomics}

Another component to account for when considering patient satisfaction and optimal treatment is the economic burden of the agent's direct and indirect costs on the patient and health system. Numerous cost-effectiveness studies have been completed to assess the burden of these newer agents compared to warfarin. Using data from clinical trials and a modeled population of 70-year-old patients with NVAF, Harrington et al estimated the cost-effectiveness of apixaban $5 \mathrm{mg}$, dabigatran $150 \mathrm{mg}$, and rivaroxaban $20 \mathrm{mg}$ compared to warfarin for stroke prevention. ${ }^{45}$ The authors found that all DOACs studied were cost-effective compared to warfarin.

\section{Conclusion}

It is well understood that DOACs have a more predictable PK profile compared to their OAC predecessor warfarin. While many clinicians are comfortable utilizing and managing DOACs in their patients, they do not come without risks and consideration for special populations, particularly those with renal impairment. Though DOACs exhibit varying degrees of renal elimination, there is a clear relationship between increased risk of bleeding and declining renal function. Based on the findings reported in this review, clinicians need to consider the patient carefully when selecting oral anticoagulation. It appears that rivaroxaban, when dosed appropriately and used judiciously, may be best in renally impaired patient. It is imperative that an assessment of renal function be based on patient-specific factors (eg, age, body mass, comorbid conditions, hydration status) and not solely on calculated $\mathrm{CrCl}$. In addition to the paramount importance of choosing therapy that is both safe and efficacious, providers also need to take patient preference into consideration. Such factors as cost, dosing frequency, monitoring, and interactions can affect how adherent patients will be to the regimen and their satisfaction with the care being provided.

\section{Disclosure}

The authors report no conflicts of interest in this work.

\section{References}

1. Pradaxa [package insert]. Ridgefield, CT: Boehringer Ingelheim Pharmaceuticals Inc; 2015

2. Xarelto [package insert]. Titusville, NJ: Janssen Pharmaceuticals Inc; 2016.

3. Eliquis [package insert]. Princeton, NJ: Bristol-Myers Squibb; 2016.

4. Savaysa [package insert]. Parsippany, NJ: Daiichi Sankyo Inc; 2015.

5. Go AS, Fang MC, Udaltsova N, et al. Impact of proteinuria and glomerular filtration rate on risk of thromboembolism in atrial fibrillation: the anticoagulation and risk factors in atrial fibrillation (ATRIA) study. Circulation. 2009;119(10):1363-1369.

6. Stangier J, Rathgen K, Stähle H, Mazur D. Influence of renal impairment on the pharmacokinetics and pharmacodynamics of oral dabigatran etexilate: an open-label, parallel-group, single-centre study. Clin Pharmacokinet. 2010;49(4):259-268.

7. Lehr T, Haertter S, Liesenfeld KH, et al. Dabigatran etexilate in atrial fibrillation patients with severe renal impairment: dose identification using pharmacokinetic modeling and simulation. J Clin Pharmacol.2012 52(9):1373-1378.

8. Kubitza D, Becka M, Mueck W, et al. Effects of renal impairment on the pharmacokinetics, pharmacodynamics and safety of rivaroxaban, an oral, direct factor Xa inhibitor. Br J Clin Pharmacol. 2010;70(5):703-712.

9. Patel MR, Mahaffey KW, Garg J, et al. Rivaroxaban versus warfarin in nonvalvular atrial fibrillation. $N$ Engl J Med. 2011;365(10):883-891.

10. EINSTEIN Investigators. Oral rivaroxaban for symptomatic venous thromboembolism. N Engl J Med. 2010;363(26):2499-2510.

11. Connolly SJ, Eikelboom J, Joyner C, et al. Apixaban in patients with atrial fibrillation. N Engl J Med. 2011;364(9):806-817.

12. Granger CB, Alexander JH, McMurray JJ, et al. Apixaban versus warfarin in patients with atrial fibrillation. $N$ Engl J Med. 2011; 365(11):981-992. 
13. Hohnloser SH, Hijazi Z, Thomas L, et al. Efficacy of apixaban when compared with warfarin in relation to renal function in patients with atrial fibrillation: insights from the ARISTOTLE trial. Eur Heart J. 2012;33(22):2821-2830.

14. Hokusai VTE Investigators. Edoxaban versus warfarin for the treatment of symptomatic venous thromboembolism. $N$ Engl J Med. 2013;369(15):1406-1415.

15. Connolly SJ, Ezekowitz MD, Yusuf S, et al. Dabigatran versus warfarin in patients with atrial fibrillation. $N$ Engl J Med. 2009;361(12): 1139-1151.

16. Hijazi Z, Hohnloser SH, Oldgren J, et al. Efficacy and safety of dabigatran compared with warfarin in relation to baseline renal function in patients with atrial fibrillation: a RE-LY (randomized evaluation of long-term anticoagulation therapy) trial analysis. Circulation. 2014; 129(9):961-970.

17. Böhm M, Ezekowitz MD, Connolly SJ, et al. Changes in renal function in patients with atrial fibrillation: an analysis from the RE-LY trial. J Am Coll Cardiol. 2015;65(23):2481-2493.

18. Fountzilas C, George J, Levine R. Dabigatran overdose secondary to acute kidney injury and amiodarone use. $N$ Z Med J. 2013;126(1370): 110-112.

19. Kernan L, Ito S, Shirazi F, Boesen K. Fatal gastrointestinal hemorrhage after a single dose of dabigatran. Clin Toxicol (Phila). 2012; 50(7):571-573.

20. Maddry JK, Amir MK, Sessions D, Heard K. Fatal dabigatran toxicity secondary to acute renal failure. Am J Emerg Med. 2013;31(2): 462.e1-e2.

21. Marino KK, Santiago RA, Dew RB 3rd, et al. Management of dabigatran-associated bleeding with two doses of idarucizumab plus hemodialysis. Pharmacotherapy. 2016;36(10):e160-e165.

22. Shafi ST, Negrete H, Roy P, Julius CJ, Sarac E. A case of dabigatranassociated acute renal failure. WMJ. 2013;112(4):173-176.

23. Stöllberger C, Lindner K, Finsterer J. Gastrointestinal bleeding under dabigatran. J Postgrad Med. 2014;60(2):192-193.

24. Wychowski MK, Kouides PA. Dabigatran-induced gastrointestinal bleeding in an elderly patient with moderate renal impairment. Ann Pharmacother. 2012;46(4):e10.

25. Samama CM, Rosencher N, Kleine E, et al. Observational study of dabigatran etexilate $150 \mathrm{mg}$ in patients with moderate renal impairment undergoing elective total hip or knee replacement. Thromb Res. 2016;143:103-110.

26. Turpie AG, Haas S, Kreutz R, et al. A non-interventional comparison of rivaroxaban with standard of care for thromboprophylaxis after major orthopaedic surgery in 17,701 patients with propensity score adjustment. Thromb Haemost. 2014;111(1):94-102.

27. Fordyce CB, Hellkamp AS, Lokhnygina Y, et al. On-treatment outcomes in patients with worsening renal function with rivaroxaban compared with warfarin: insights from ROCKET AF. Circulation. 2016; 134(1):37-47.

28. Hori M, Matsumoto M, Tanahashi N, et al. Rivaroxaban vs. warfarin in Japanese patients with non-valvular atrial fibrillation in relation to age. Circ J. 2014;78(6):1349-1356.

29. Suzuki M, Fukamizu S, Oyama J, et al. Rationale and design of the efficacy of rivaroxaban on renal function in patients with non-valvular atrial fibrillation and chronic kidney disease: the X-NOAC study. Int $J$ Cardiol. 2015;188:52-53.

Therapeutics and Clinical Risk Management

\section{Publish your work in this journal}

Therapeutics and Clinical Risk Management is an international, peerreviewed journal of clinical therapeutics and risk management, focusing on concise rapid reporting of clinical studies in all therapeutic areas, outcomes, safety, and programs for the effective, safe, and sustained use of medicines. This journal is indexed on PubMed Central, CAS,
30. Stöllberger C, Finsterer J. Rivaroxaban $10 \mathrm{mg} / \mathrm{d}$ in severe renal failure does not prevent ischemic events in premorbid neurologic disease. Neurol Neurochir Pol. 2015;49(3):180-182.

31. Kufel WD, Zayac AS, Lehmann DF, Miller CD. Clinical application and pharmacodynamic monitoring of apixaban in a patient with endstage renal disease requiring chronic hemodialysis. Pharmacotherapy. 2016;36(11):e166-e171.

32. Eikelboom JW, Connolly SJ, Gao P, et al. Stroke risk and efficacy of apixaban in atrial fibrillation patients with moderate chronic kidney disease. J Stroke Cerebrovasc Dis. 2012;21(6):429-435.

33. Koretsune Y, Yamashita T, Kimura T, Fukuzawa M, Abe K, Yasaka M. Short-term safety and plasma concentrations of edoxaban in Japanese patients with non-valvular atrial fibrillation and severe renal impairment. Circ J. 2015;79(7):1486-1495.

34. Coleman CI, Limone B, Sobieraj DM, et al. Dosing frequency and medication adherence in chronic disease. J Manag Care Pharm. 2012;18(7): 527-539.

35. Coleman CI, Roberts MS, Sobieraj DM, Lee S, Alam T, Kaur R. Effect of dosing frequency on chronic cardiovascular disease medication adherence. Curr Med Res Opin. 2012;28(5):669-680.

36. Claxton AJ, Cramer J, Pierce C. A systematic review of the associations between dose regimens and medication compliance. Clin Ther. 2001;23(8):1296-1310.

37. Shore S, Carey EP, Turakhia MP, et al. Adherence to dabigatran therapy and longitudinal patient outcomes: insights from the Veterans Health Administration. Am Heart J. 2014;167(6):810-817.

38. Coumadin [package insert]. Princeton, NJ: Bristol-Myers Squibb; 2011.

39. Attaya S, Bornstein T, Ronquillo N, et al. Study of warfarin patients investigating attitudes toward therapy change (SWITCH survey). Am J Ther. 2012;19(6):432-435.

40. Elewa HF, DeRemer CE, Keller K, Gujral J, Joshua TV. Patients satisfaction with warfarin and willingness to switch to dabigatran: a patient survey. J Thromb Thrombolysis. 2014;38(1):115-120.

41. Ghijben P, Lancsar E, Zavarsek S. Preferences for oral anticoagulants in atrial fibrillation: a best-best discrete choice experiment. Pharmacoeconomics. 2014;32(11):1115-1127.

42. Coleman CI, Coleman SM, Vanderpoel J, et al. Patient satisfaction with warfarin- and non-warfarin-containing thromboprophylaxis regimens for atrial fibrillation. J Investig Med. 2013;61(5):878-881.

43. Monz BU, Connolly SJ, Korhonen M, Noack H, Pooley J. Assessing the impact of dabigatran and warfarin on health-related quality of life: results from an RE-LY sub-study. Int J Cardiol. 2013;168(3):2540-2547.

44. Camm AJ, Amarenco P, Haas S, et al. XANTUS: a real-world, prospective, observational study of patients treated with rivaroxaban for stroke prevention in atrial fibrillation. Eur Heart J. 2016;37(14): 1145-1153.

45. Harrington AR, Armstrong EP, Nolan PE Jr, Malone DC. Costeffectiveness of apixaban, dabigatran, rivaroxaban, and warfarin for stroke prevention in atrial fibrillation. Stroke. 2013;44(6):1676-1681.

46. Stacy ZA, Call WB, Harmann AP, Peters GL, Richter SK. Edoxaban: a comprehensive review of the pharmacology and clinical data for the management of atrial fibrillation and venous thromboembolism. Cardiol Ther. 2016:5;1-18.

EMBase, Scopus and the Elsevier Bibliographic databases. The manuscript management system is completely online and includes a very quick and fair peer-review system, which is all easy to use. Visit http://www.dovepress.com/testimonials.php to read real quotes from published authors. 